\title{
Hyperfine Structure and Relaxation Times of 4-Oxo-TEMPO/Methyl Alcohol Solutions in Weak and Strong Fields
}

\author{
M. Sünnetçioğlu, G. Bingöl, and R. Sungur \\ Magnetic Resonance Laboratory, Department of Physics Engineering, Faculty of Engineering, \\ Hacettepe University, Beytepe-Ankara, Turkey
}

Z. Naturforsch. 46a, 976-982 (1991); received July 24, 1991

The hyperfine structure of the 4-Oxo-TEMPO (2,2,6,6-tetramethyl-4-oxopiperidin-oxyl-1) free radical was investigated. Theoretical investigation includes four methyl groups protons close to the unpaired electron. Energy levels and transition probabilities were found by using second order and first order perturbation theory, respectively, and from these data theoretical spectra of the free radical were obtained. Diluted solutions in $\mathrm{CH}_{3}-\mathrm{OH}$ were prepared and the spectra were recorded with a double resonance spectrometer $(1.53 \mathrm{mT})$ and Varian E-9 X-band ESR spectrometer $(\sim 0.3 \mathrm{~T})$. In order to understand the influence of time dependent phenomena on the linewidths, electronic relaxation times of 4-Oxo-TEMPO and its perdeuterated form (PDT) were measured at two different fields by using cw saturation method.

\section{Introduction}

Studies of nitroxide free radicals by NMR were usually restricted to the strong field region. In the present work on methyl alcohol solutions of 4-OxoTEMPO free radical (Fig. 1) and its perdeuterated form we included the weak field region. Our first aim was to obtain theoretical spectra in weak and strong fields and compare them with the experimental ones. Therefore, starting from the spin Hamiltonian and using perturbation theory, energy eigenvalues, eigenfunctions, transition probabilities, energy level broadenings and resonance frequencies (or resonance fields) were calculated and theoretical spectra of the free radical were thus obtained.

Electronic relaxation time measurements on each nitrogen hyperfine line are necessary in order to determine the effect of time dependent phenomena on the linewidths. Therefore such measurements on each nitrogen hyperfine line, at weak and strong field are selected as the second aim of this study.

\section{Theory}

The time independent effect of the molecular protons was calculated using a model composed of $1 \mathrm{e}^{-}$,

Reprint requests to Dr. M. Sünnetçioğlu, Hacettepe Üniversitesi, Mühendislik Fakültesi, Fizik Mühendisliği Bölümü, 06532 Beytepe-Ankara, Turkey.
$1 \mathrm{~N}$ and 12 equivalent protons. The Hamiltonian of the system is given by [1]

$$
\begin{aligned}
\mathscr{H}= & g \beta B_{0} S_{z}+A_{1} S_{z} I_{N z}+A_{2} S_{z} I_{H z} \\
& +\left(A_{1} / 2\right)\left\{S^{+} I_{N}^{-}+S^{-} I_{N}^{+}\right\}+\left(A_{2} / 2\right)\left\{S^{+} I_{H}^{-}+S^{-} I_{H}^{+}\right\},
\end{aligned}
$$

where

$$
I_{H}=\sum_{i} I_{H i}, \quad i=1,2, \ldots, 12 .
$$

By constructing the Hamiltonian matrix, which has the dimension $294 \times 294$ and consists of thirty $6 \times 6$, twelve $5 \times 5$, twelve $3 \times 3$, two $2 \times 2$ and fourteen $1 \times 1$ submatrices, and treating the last term in (1) as perturbation, energy eigenvalues and eigenfunctions were calculated using second and first order perturbation theory, respectively. The general matrix of perturbation $\left(\mathscr{H}_{1}\right)$ for a submatrix which has $6 \times 6$ or smaller dimension is given in Table 1. In this table and in the following calculations $i$ represents the first element of the submatrix. If the perturbation term is not taken

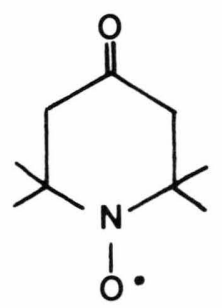

Fig. 1. 4-Oxo-TEMPO (2,2,6,6-tetramethyl-4-oxopiperidinoxyl-1) free radical. 
Table 1. General matrix of perturbation.

\begin{tabular}{|c|c|c|c|c|c|c|}
\hline $\mathscr{H}_{1}$ & $\left|\psi_{i}\right\rangle^{0}$ & $\left|\psi_{i+1}\right\rangle^{0}$ & $\left|\psi_{i+2}\right\rangle^{0}$ & $\left|\psi_{i+3}\right\rangle^{0}$ & $\left|\psi_{i+4}\right\rangle^{0}$ & $\left|\psi_{i+5}\right\rangle^{0}$ \\
\hline${ }^{0}\left\langle\psi_{i}\right|$ & 0 & $A_{2} C_{1} a_{i+1}$ & $-A_{2} C_{1} a_{i+2}$ & 0 & 0 & 0 \\
\hline${ }^{0}\left\langle\psi_{i+1}\right|$ & $A_{2} C_{1} a_{i+1}$ & 0 & 0 & $A_{2} C_{2} a_{i+2} a_{i+3}$ & $-A_{2} C_{2} a_{i+2} a_{i+4}$ & 0 \\
\hline${ }^{0}\left\langle\psi_{i+2}\right|$ & $-A_{2} C_{1} a_{i+2}$ & 0 & 0 & $A_{2} C_{2} a_{i+1} a_{i+3}$ & $-A_{2} C_{2} a_{i+1} a_{i+4}$ & 0 \\
\hline${ }^{0}\left\langle\psi_{i+3}\right|$ & 0 & $A_{2} C_{2} a_{i+2} a_{i+3}$ & $A_{2} C_{2} a_{i+1} a_{i+3}$ & 0 & 0 & $A_{2} C_{3} a_{i+4}$ \\
\hline $0<\psi_{i+4}$ & 0 & $-A_{2} C_{2} a_{i+2} a_{i+4}$ & $-A_{2} C_{2} a_{i+1} a_{i+4}$ & 0 & 0 & $A_{2} C_{3} a_{i+3}$ \\
\hline${ }^{0}\left\langle\psi_{i+5}\right|$ & 0 & 0 & & $A_{2} C_{3} a_{i+4}$ & $A_{2} C_{3} a_{i+3}$ & \\
\hline
\end{tabular}

into account, the eigenfunctions of the system for an arbitrary $6 \times 6$ submatrix are

$$
\begin{aligned}
& \left|\psi_{i}\right\rangle^{0}=|K\rangle_{i}, \\
& \left|\psi_{i+1}\right\rangle^{0}=a_{i+1}|K\rangle_{i+1}+a_{i+2}|K\rangle_{i+2}, \\
& \left|\psi_{i+2}\right\rangle^{0}=-a_{i+2}|K\rangle_{i+1}+a_{i+1}|K\rangle_{i+2}, \\
& \left|\psi_{i+3}\right\rangle^{0}=a_{i+3}|K\rangle_{i+3}+a_{i+4}|K\rangle_{i+4}, \\
& \left|\psi_{i+4}\right\rangle^{0}=-a_{i+4}|K\rangle_{i+3}+a_{i+3}|K\rangle_{i+4}, \\
& \left|\psi_{i+5}\right\rangle^{0}=|K\rangle_{i+5},
\end{aligned}
$$

where $|K\rangle_{i}$ are the base vectors.

Second order corrections to the energies is given by

$$
E_{i}(\lambda)=E_{i}^{0}+\sum_{i \neq j} \frac{\left|{ }^{0}\left\langle\psi_{i}\left|\mathscr{H}_{1}\right| \psi_{j}\right\rangle^{0}\right|^{2}}{E_{i}^{0}-E_{j}^{0}} .
$$

Using (3) and the general perturbation matrix in Table 1, the energy eigenvalues were obtained

$$
\begin{gathered}
E_{i}=E_{i}^{0}+\frac{A_{2}^{2} C_{1}^{2} a_{i+1}^{2}}{E_{i}^{0}-E_{i+1}^{0}}+\frac{A_{2}^{2} C_{1}^{2} a_{i+2}^{2}}{E_{i}^{0}-E_{i+2}^{0}}, \\
E_{i+1}=E_{i+1}^{0}+\frac{A_{2}^{2} C_{1}^{2} a_{i+1}^{2}}{E_{i+1}^{0}-E_{i}^{0}}+\frac{A_{2}^{2} C_{2}^{2} a_{i+2}^{2} a_{i+3}^{2}}{E_{i+1}^{0}-E_{i+3}^{0}} \\
+\frac{A_{2}^{2} C_{2}^{2} a_{i+2}^{2} a_{i+4}^{2}}{E_{i+1}^{0}-E_{i+4}^{0}}, \\
E_{i+2}=E_{i+2}^{0}+\frac{A_{2}^{2} C_{1}^{2} a_{i+2}^{2}}{E_{i+2}^{0}-E_{i}^{0}}+\frac{A_{2}^{2} C_{2}^{2} a_{i+1}^{2} a_{i+3}^{2}}{E_{i+2}^{0}-E_{i+3}^{0}} \\
+\frac{A_{2}^{2} C_{2}^{2} a_{i+1}^{2} a_{i+4}^{2}}{E_{i+2}^{0}-E_{i+4}^{0}}, \\
E_{i+3}=E_{i+3}^{0}+\frac{A_{2}^{2} C_{3}^{2} a_{i+4}^{2}}{E_{i+3}^{0}-E_{i+5}^{0}}+\frac{A_{2}^{2} C_{2}^{2} a_{i+1}^{2} a_{i+3}^{2}}{E_{i+3}^{0}-E_{i+2}^{0}} \\
+\frac{A_{2}^{2} C_{2}^{2} a_{i+2}^{2} a_{i+3}^{2}}{E_{i+3}^{0}-E_{i+1}^{0}},
\end{gathered}
$$

$$
E_{i+4}=E_{i+4}^{0}+\frac{A_{2}^{2} C_{3}^{2} a_{i+3}^{2}}{E_{i+4}^{0}-E_{i+5}^{0}}+\frac{A_{2}^{2} C_{2}^{2} a_{i+1}^{2} a_{i+4}^{2}}{E_{i+4}^{0}-E_{i+2}^{0}}
$$

$$
+\frac{A_{2}^{2} C_{2}^{2} a_{i+2}^{2} a_{i+4}^{2}}{E_{i+4}^{0}-E_{i+1}^{0}}
$$

$$
E_{i+5}=E_{i+5}^{0}+\frac{A_{2}^{2} C_{3}^{2} a_{i+4}^{2}}{E_{i+5}^{0}-E_{i+3}^{0}}+\frac{A_{2}^{2} C_{3}^{2} a_{i+3}^{2}}{E_{i+5}^{0}-E_{i+4}^{0}} .
$$

Using first order perturbation, the eigenfunctions take the form

$$
\left|\psi_{i}(\lambda)\right\rangle=\left|\psi_{i}\right\rangle^{0}+\sum_{i \neq j} \frac{\left.\right|^{0}\left\langle\psi_{j}\left|\mathscr{H}_{1}\right| \psi_{i}\right\rangle^{0} \mid}{E_{i}^{0}-E_{j}^{0}}\left|\psi_{j}\right\rangle^{0} .
$$

Eigenfunctions for an arbitrary $6 \times 6$ submatrix were obtained using (5) and are listed in Table 2 . In this table $X_{j}(j=i, \ldots, i+5)$ are the normalization coefficients and $W_{k j}(k=1, \ldots, 6 ; j=i, \ldots, i+5)$ are the coefficients of the eigenfunctions, which are generalized as in Table 3.

The general equation for the probabilities of the transitions between energy levels of the system reads

$$
\begin{aligned}
P_{m n}=C\left|\frac{W_{1 m} W_{2 n}+W_{3 m} W_{4 n}+W_{5 m} W_{6 n}}{2 X_{m} X_{n}}\right|^{2}, \\
m=1, \ldots, 294 ; n=m+1, \ldots, 294 .
\end{aligned}
$$

From these relations, with the aid of a computer, energy eigenvalues and transition probabilities were calculated and plotted as functions of the magnetic field. Figure $2 \mathrm{a}, \mathrm{b}$ show that the presence of protons causes unequal and equal energy level broadenings in the weak and strong magnetic fields, respectively.

The transition probabilities of the system form 8 main groups [2], each group including 85 transitions. Since the values within a group are very close to each other, only one transition probability is selected from each group and plotted as a function of the magnetic field in Figure 3. 

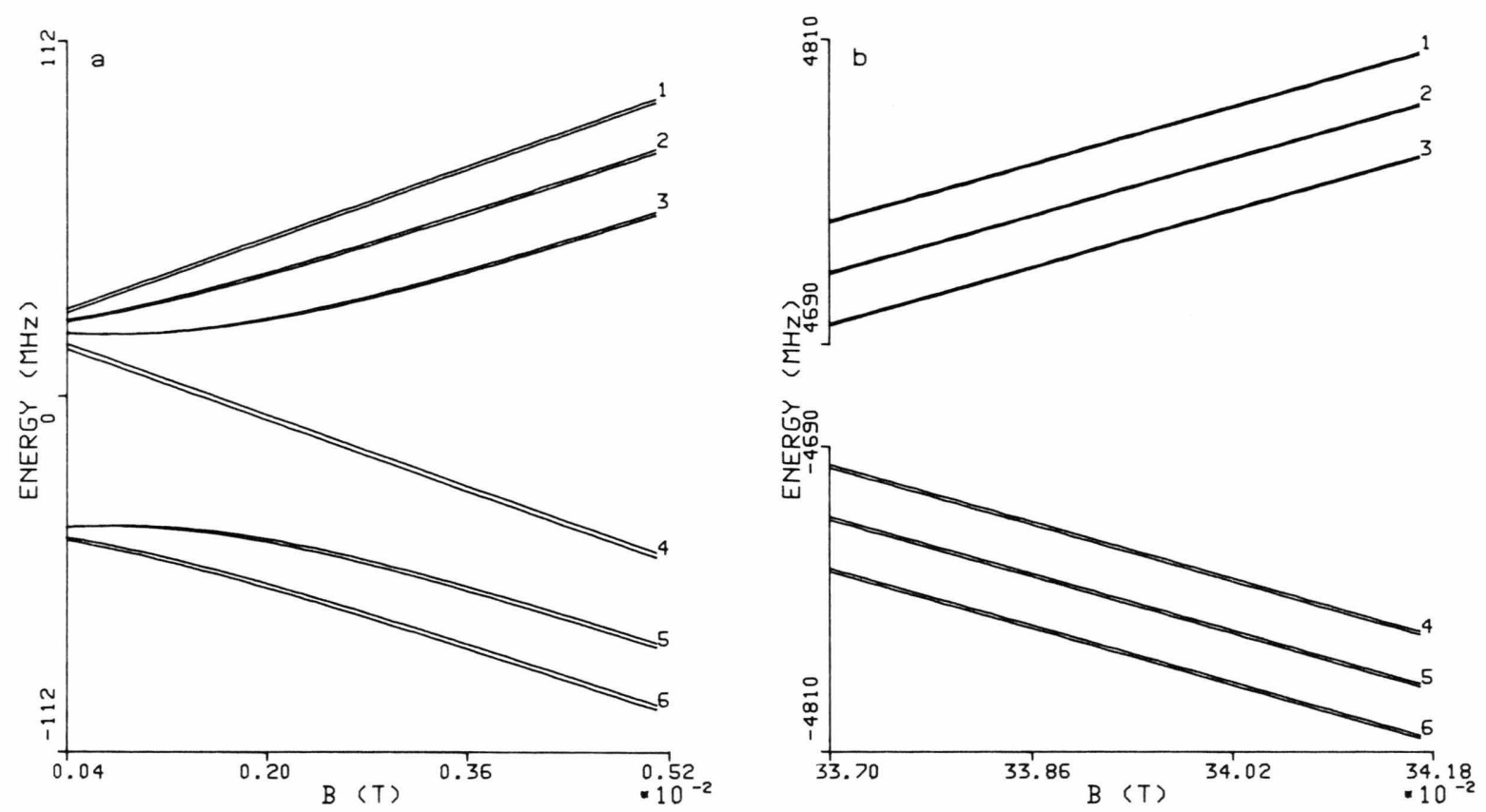

Fig. 2. Energy levels of the $1 \mathrm{e}^{-}, 1 \mathrm{~N}, 12 \mathrm{p}$ system: a) weak field, b) strong field.

Table 2. Generalized eigenfunctions.

$$
\begin{aligned}
\left|\psi_{i}\right\rangle & =\frac{1}{X_{i}}\left\{W_{1 i}|K\rangle_{i}+W_{2 i}|K\rangle_{i+1}+W_{3 i}|K\rangle_{i+2}\right\} \\
\left|\psi_{i+1}\right\rangle & =\frac{1}{X_{i+1}}\left\{W_{1 i+1}|K\rangle_{i}+W_{2 i+1}|K\rangle_{i+1}+W_{3 i+1}|K\rangle_{i+2}+W_{4 i+1}|K\rangle_{i+3}+W_{5 i+1}|K\rangle_{i+4}\right\} \\
\left|\psi_{i+2}\right\rangle & =\frac{1}{X_{i+2}}\left\{W_{1 i+2}|K\rangle_{i}+W_{2 i+2}|K\rangle_{i+1}+W_{3 i+2}|K\rangle_{i+2}+W_{4 i+2}|K\rangle_{i+3}+W_{5 i+2}|K\rangle_{i+4}\right\} \\
\left|\psi_{i+3}\right\rangle & =\frac{1}{X_{i+3}}\left\{W_{2 i+3}|K\rangle_{i+1}+W_{3 i+3}|K\rangle_{i+2}+W_{4 i+3}|K\rangle_{i+3}+W_{5 i+3}|K\rangle_{i+4}+W_{6 i+3}|K\rangle_{i+5}\right\} \\
\left|\psi_{i+4}\right\rangle & =\frac{1}{X_{i+4}}\left\{W_{2 i+4}|K\rangle_{i+1}+W_{3 i+4}|K\rangle_{i+2}+W_{4 i+4}|K\rangle_{i+3}+W_{5 i+4}|K\rangle_{i+4}+W_{6 i+4}|K\rangle_{i+5}\right\} \\
\left|\psi_{i+5}\right\rangle & =\frac{1}{X_{i+5}}\left\{W_{4 i+5}|K\rangle_{i+3}+W_{5 i+5}|K\rangle_{i+4}+w_{6 i+5}|K\rangle_{i+5}\right\}
\end{aligned}
$$

\section{Experimental}

Diluted solutions $\left(10^{-3} \mathrm{M}\right)$ of 4-Oxo-TEMPO and PDT in methyl alcohol were deoxygenated in a vacuum line by repeated freeze-pump-thaw technique. The samples were sealed off under a pressure of $10^{-5}$ $\mathrm{mm} \mathrm{Hg}$. All the measurements were performed at $293 \mathrm{~K}$.

\section{a) Weak Field}

Weak field measurements were made on a homemade nuclear-electron double resonance spectrometer, based on Overhauser effect [3]. In this spectrometer, dynamic nuclear polarization $P_{z}$ can be measured at different points on the ESR spectrum [4]. In obtaining the ESR spectra, the magnetic field $(1.53 \mathrm{mT})$ and 


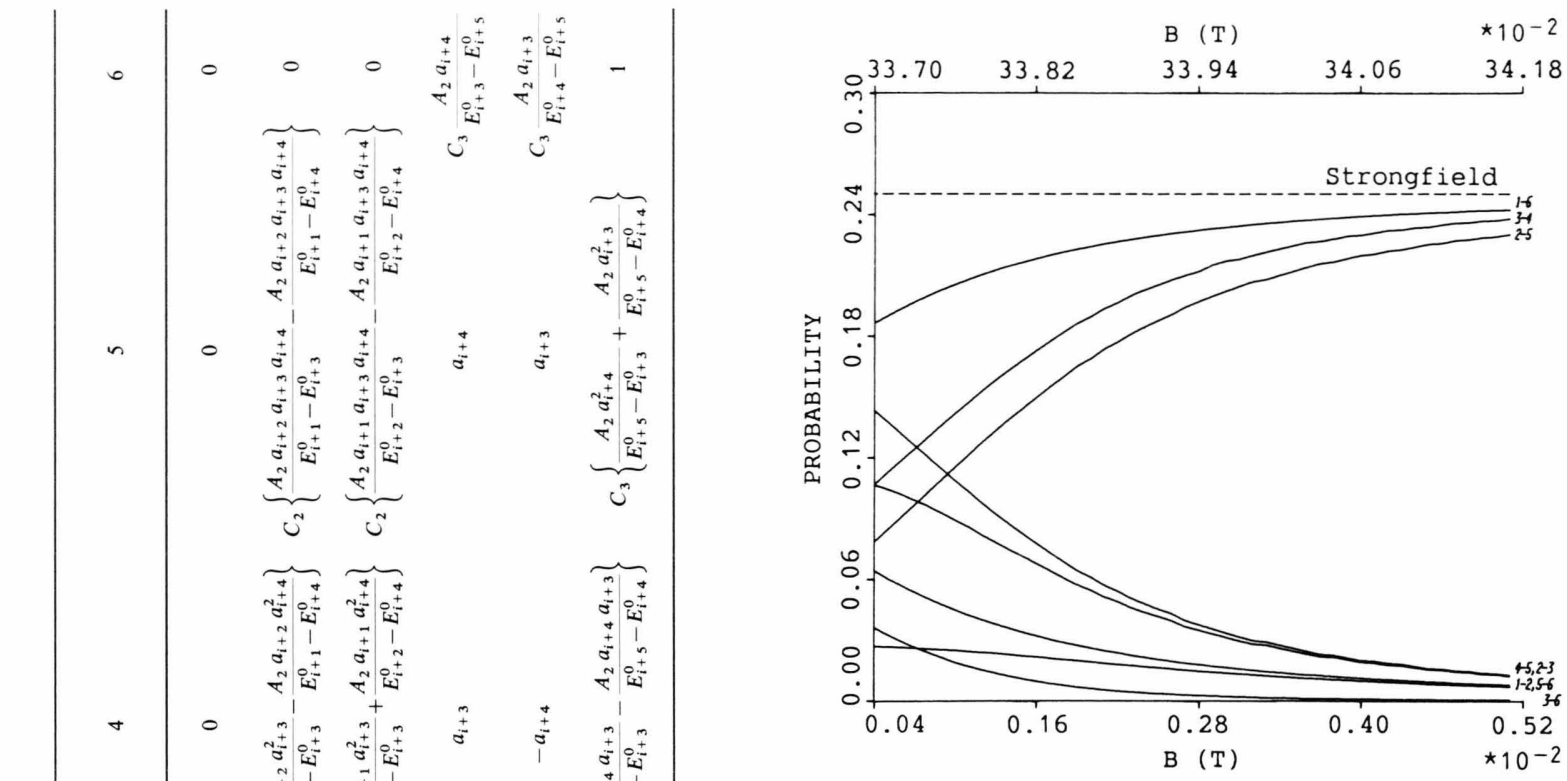

Fig. 3. Transition probabilities of the $1 \mathrm{e}^{-}, 1 \mathrm{~N}, 12 \mathrm{p}$ system.

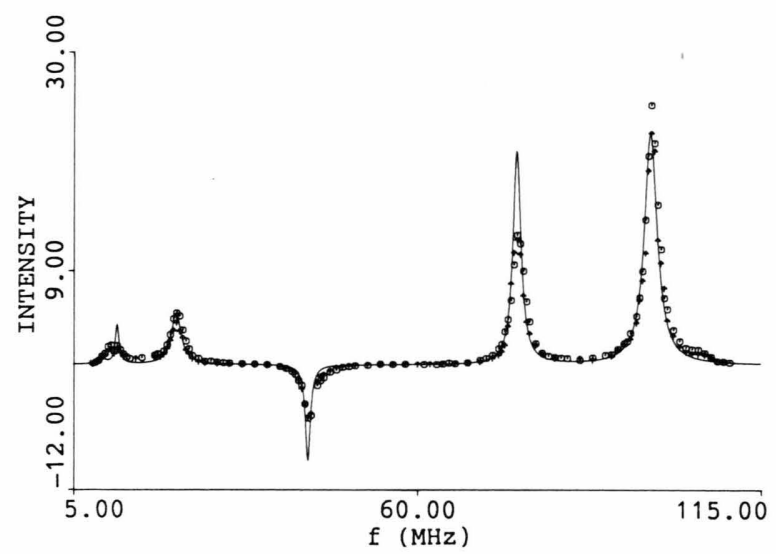

Fig. 4. Weak field spectra of 4-Oxo-TEMPO, PDT/methyl alcohol. — Theoretical, experimental ( $\_$४ 4-Oxo-TEMPO, $\odot \odot$ PDT).

the nuclear resonance frequency $(65 \mathrm{kHz})$ are kept constant and the ESR frequency is varied in steps keeping the ESR field amplitude unchanged. By measuring enhanced nuclear polarizations $P_{z}$ in this way and the nuclear polarization $P_{0}$ in the absence of ESR power, observed enhancements $G(P)=\left(P_{z}-P_{0}\right) / P_{0}$ were calculated. By plotting $G(P)$ as a function of the ESR frequency we obtained the experimental spectrum in Figure 4. In this spectrum, $P_{z}$ and $P_{0}$ have opposite signs for all transitions except the one at $42.6 \mathrm{MHz}$. 
Generally, as a result of the Overhauser effect, the nuclear spin system emits radiation at ESR frequencies whereas the electronic spin system absorbs the energy. In another words, there are more nuclear spins in the higher energy level than in the lower one. For the transition $4-5(42.6 \mathrm{MHz})$ the situation is opposite and the nuclear spin system absorbs radiation. Accordingly this resonance line is in the opposite direction.

Electronic relaxation times were measured by the $\mathrm{cw}$ saturation method using dynamic nulear polarization (DNP) technique $[4,5]$. As a result of the presence of the nitrogen nucleus in the molecular structure, weak field experimental spectra of nitroxides consist of 6 well resolved resonance lines. These lines must be saturated separately if the cw saturation method is used in the determination of electronic relaxation times. In this case, the saturation parameter $s$ is given by

$$
s=\sum_{i j} s_{i j}=\sum_{i j} \frac{\sigma_{i j} g\left(\omega-\omega_{i j}\right)}{1+\sigma_{i j} g\left(\omega-\omega_{i j}\right)} h\left(\omega_{i j}\right) .
$$

Here $g\left(\omega-\omega_{i j}\right)$ is the shape function, $h\left(\omega_{i j}\right)$ is the weighting factor which depends on the electronic relaxation process and

$$
\sigma_{i j} \equiv \pi \gamma_{s}^{2} B_{1}^{2} T_{1}^{i j}
$$

If $\sigma_{i j} g\left(\omega-\omega_{i j}\right) \ll 1, s_{i j}$ can be rewritten as

$$
s_{i j}=\sigma_{i j} g\left(\omega-\omega_{i j}\right) h\left(\omega_{i j}\right) \text {. }
$$

The observed enhancement for $i j$-th transition is [6]

$G(P)=\left(P_{z}-P_{0}\right) / P_{0}=-\varrho f\left(\omega_{s} / \omega_{\mathrm{I}}\right) s_{i j}=-F_{i j} s_{i j}$,

where $\varrho$ is the nucleus-electron coupling parameter, $f$ is the leakage factor, and $\omega_{s}$ and $\omega_{\mathrm{I}}$ are electronic and nuclear resonance frequencies, respectively. In previous studies done at $1.53 \mathrm{mT}$, the ratio of $\gamma_{s} / \gamma_{\mathrm{I}}$ was used instead of $\omega_{s} / \omega_{\mathrm{I}}$ in $(10)[2,5]$. This approximation can be made only if the experiment could be performed at the centre of ESR and NMR lines simultaneously [6-8]. In this study, the observed ESR frequencies are

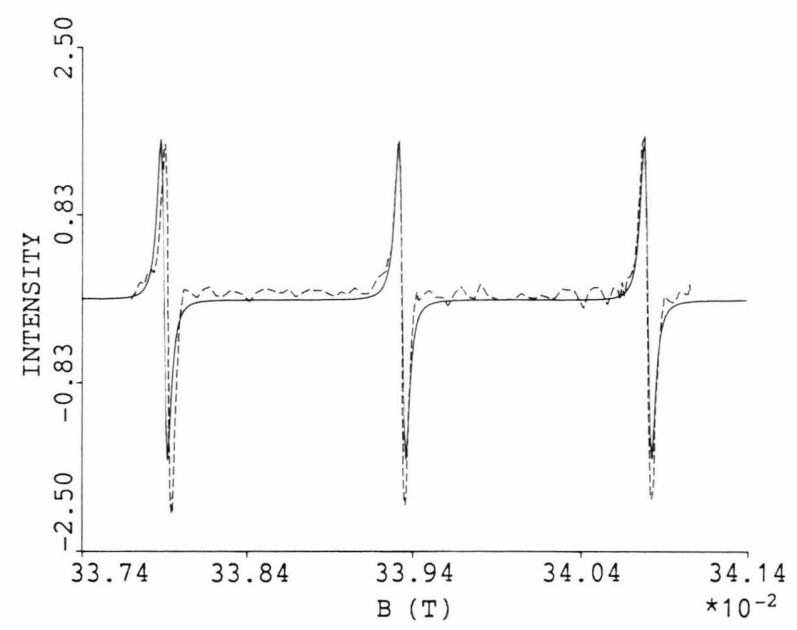

Fig. 5. Strong field spectra of 4-Oxo-TEMPO/methyl alcohol. Theoretical, --- experimental.

spread over a large frequency band $(\approx 90 \mathrm{MHz})$ and accordingly the observed enhancements are firmly effected by the $\omega_{s} / \omega_{\mathrm{I}}$ ratios.

According to (8), (9), (10), the electronic relaxation times are calculated from the relation

$$
T_{1}^{i j}=\frac{\left|\left(P_{z}-P_{0}\right) / P_{0}\right|_{\text {low power }}}{F_{i j} h\left(\omega_{i j}\right)} \frac{1}{\pi \gamma_{s}^{2} B_{1}^{2} g\left(\omega-\omega_{i j}\right)} .
$$

Since $s_{i j} \propto B_{1}^{2} \propto V^{2}\left(B_{1}\right.$ is the rf field amplitude on the sample and $V$ is the applied voltage to establish this field), the graphs $|G(P)|^{-1}=f\left(V^{-2}\right)$ are straight lines. The coefficients $F_{i j} h\left(\omega_{i j}\right)$ are obtained by extrapolating these lines. By using the low power $G(P)$ value, the saturation factor $s_{i j}$ for the $i j$-th resonance line can be determined. Since the peaks at $11 \mathrm{MHz}$ and $12 \mathrm{MHz}$ were not completely resolved, a common saturation parameter was calculated for these peaks. The measured relaxation times are given in Table 4.

\section{b) Strong Field}

Strong field spectra were recorded with a Varian

\begin{tabular}{|c|c|c|c|c|c|c|c|c|c|}
\hline \multirow{2}{*}{$\begin{array}{l}\text { Resonance } \\
\text { field (mT) } \\
m_{1}\end{array}$} & \multicolumn{6}{|c|}{ Weak field } & \multicolumn{3}{|c|}{ Strong field } \\
\hline & 3.93 & 4.28 & 7.68 & 15.18 & 27.14 & 34.82 & $\begin{array}{l}337.92 \\
(+1)\end{array}$ & $\begin{array}{l}339.34 \\
(0)\end{array}$ & $\begin{array}{l}340.81 \\
(-1)\end{array}$ \\
\hline 4-Охо-ТЕМРO & \multicolumn{2}{|c|}{0.21} & 1.02 & 1.60 & 1.89 & 2.76 & 1.10 & 0.99 & 0.99 \\
\hline PDT & \multicolumn{2}{|c|}{0.14} & 1.00 & 0.75 & 1.06 & 2.00 & 1.18 & 1.06 & 1.25 \\
\hline
\end{tabular}
E-9X-band spectrometer. The experimental spectrum

Table 4. Electronic relaxation times of 4-Oxo-TEMPO/methyl alcohol and PDT/methyl alcohol at weak and strong field $\left(T_{1} \times 10^{7} \mathrm{~s}\right)$. 
is given in Figure 5. The cw saturation method was also used in the relaxation time measurements, in this field.

The relaxation time is given by

$$
T_{1}=\left(\frac{3^{1 / 2} \Delta B_{\mathrm{pp}}^{0}}{2 \gamma}\right)\left(\frac{1 / s-1}{B_{1}^{2}}\right) .
$$

Here $\Delta B_{\mathrm{pp}}^{0}$ is the unsaturated peak-to-peak linewidth, $s$ the saturation factor, and $B_{1}$ the microwave amplitude. $B_{1}$ is given by

$$
B_{1}^{2}=\frac{2 B_{0}^{2} \mu}{4 \pi}\left(1-\left|\Gamma_{0}\right|^{2}\right) \frac{P_{\text {inc }} Q}{v_{0}} .
$$

Here $B_{0}^{2}$ is the reduced magnetic field parameter, which was measured by the perturbing spheres technique [9], $P_{\text {inc }}$ is the incident power at the cavity, $\mu$ the magnetic permeability, $\Gamma_{0}$ the cavity voltage reflection coefficient, $Q$ the loaded cavity quality factor, and $v_{0}$ the resonance frequency. The cavity quality factors were measured by using a crystal detector. The results are listed in Table 4.

\section{Results and Discussion}

\section{a) Weak Field}

According to the theoretical results, transitions between the energy levels $1-6,2-5=3-6,2-3=5-6$, $1-2,3-4,4-5$ are possible (Figure $2 \mathrm{a}$ ). The theoretical spectrum was obtained by taking the differences between the mean values of the related energy levels as centers of resonance peaks and the energy level broadenings as linewidths. A starting value for the electron-nitrogen nucleus hyperfine coupling constant $A_{1}$ is obtained from the strong field ESR spectra and for the electron-protons hyperfine coupling constant $A_{2}$ from previous papers [10-13]. These parameters were changed in order to find the best fit between theoretical and experimental resonance frequencies. The value of $A_{2}$ mostly effects the linewidths. Because of the relaxation effects which were not included in the theoretical calculations, the theoretical linewidths must not exceed the experimental ones. In order to fulfill this requirement, the determination of $A_{2}$ needs special care.

Taking $\omega_{s} / \omega_{\mathrm{I}}$ ratios under consideration, the best fit (Fig. 4) is obtained for the following values of $A_{1}$ and $A_{2}$ :

$$
A_{1}=(42.6 \pm 0.1) \mathrm{MHz}, \quad A_{2}=(0.24 \pm 0.02) \mathrm{MHz} .
$$

The linewidths can be tested in another way. Since $\omega \tau_{\mathrm{c}} \ll 1$ at weak field, $T_{1}=T_{2}$. Therefore measurements of $T_{1}$ directly give information about linewidths. As mentioned above, theoretical linewidths must be narrower than found from $T_{1}$. This requirement is also fulfilled for all the resonance frequencies except the one at $97.5 \mathrm{MHz}$.

Figure 4 shows that there is no remarkable difference between the experimental spectra of 4-OxoTEMPO and of PDT (this is also the case in strong field). Therefore one can conclude that electronprotons interactions are very weak in the molecule. One can also arrive to this conclusion from the weak and strong field experimental spectra of 4-OxoTEMPO, which do not show proton hyperfine structure.

As shown in Table 4, the electronic relaxation times are frequency dependent. The source of this dependence is the saturation parameter. As known, the saturation parameter is a function of the population differences in the stationary state and in thermal equilibrium [2, 6]. As a result of DNP, the population differences change from peak to peak, and correspondingly the saturation parameter changes as a function of transition probability and frequency. Consequently, according to (10) and (11), the measured relaxation times differ from one hyperfine line to another.

A comparison of the relaxation times of 4-OxoTEMPO and PDT shows that the relaxation times of 4-Oxo-TEMPO, contrary to expectation, are greater than those of PDT. The reason of this may be the concentration difference between these two samples. Previous studies at weak field have shown that the primary source of relaxation is dipolar interaction between electrons and solvent protons $[5,14]$, which is proportional to concentration. This is also confirmed in this work, getting negative values for the enhancement factors. Accordingly, the concentration of PDT/ methyl alcohol is higher than that of 4-Oxo-TEMPO/ methyl alcohol.

\section{b) Strong Field}

Theoretically there may be transitions between the energy levels 1-6, 2-5, 3-4 (Figure 2b). As shown in Fig. 3 , in the strong field five of the transition probability functions diminish and the other three join at the same value. In this field region, the method to obtain the theoretical spectrum and the starting values for $A_{1}$ and $A_{2}$ is the same as in the weak field case. The 
best fit (Fig. 5) is obtained for the following values:

$A_{1}=(40.60 \pm 0.05) \mathrm{MHz}, \quad A_{2}=(0.168 \pm 0.005) \mathrm{MHz}$.

These values are smaller than those obtained at weak field. Therefore the values of $A_{1}$ and $A_{2}$ may be frequency dependent. The reason of this behaviour can be explained as follows. The hyperfine coupling constants include the amplitude of the electronic wave function on the nuclei. As the magnetic field changes from the strong to the weak field region, the correlation times change [14]. Therefore the amplitude of the electronic wave function on the nuclei may also change. Consequently, the probability of finding electron around nuclei is smaller at strong than at weak field.

[1] W. Müller-Warmuth, Z. Naturforsch. 19a, 1309 (1964).

[2] W. Müller-Warmuth, Z. Naturforsch. 15a, 927 (1960).

[3] N. Zengin and G. Bingöl, Commun. Fac. des Sciences d'Ankara 16 A, 71 (1967).

[4] J. Haupt and W. Müller-Warmuth, Z. Naturforsch. 17a, 1011 (1962).

[5] W. Müller-Warmuth, E. Öztekin, R. Vilhjalmsson, and A. Yalçiner, Z. Naturforsch. 25a, 1688 (1970).

[6] R. D. Bates, Jr. and W. S. Drozdoski, J. Chem. Phys. 67, 4038 (1977)

[7] G. J. Gerardi, B. E. Wagner, and J. A. Potenza, J. Chem. Phys. 69, 4645 (1978).

[8] R. D. Bates, Jr., J. Magn. Reson. 48, 111 (1982).

[9] R. D. Rataiczak and M. T. Jones, J. Chem. Phys. 56, $3898(1972)$
An inspection of measured relaxation times at strong field shows that the values for PDT/methyl alcohol are greater than for 4-Oxo-TEMPO/methyl alcohol. This is expected, since there are many sources, other than dipolar interactions, for electronic relaxation $[12,15,16]$, and concentration is of minor importance in this case. For the probes used, the dominant relaxation mechanism, besides the electronnucleus dipolar interactions, may be the anisotropy of the $g$-tensor modulated by rotational diffusion and spin-rotation $[16,17]$.

In free radical solutions, electronic relaxation times change for various hyperfine lines, depending upon nuclear magnetic quantum numbers [18]. This explains the differences of $T_{1}$ in strong field.

[10] R. W. Kreilick, J. Chem. Phys. 46, 4260 (1967).

[11] G. Poggi and C. S. Johnson, Jr., J. Magn. Reson. 3, 436 (1970).

[12] C. Jolicoeur and H. L. Friedman, J. Solution Chem. 3, 15 (1974).

[13] J. J. Windle, J. Magn. Reson. 45, 432 (1981).

[14] K. D. Kramer, W. Müller-Warmuth, and J. Schindler, J. Chem. Phys. 43, 31 (1965).

[15] Y. Y. Lim, E. A. Smith, and M. C. R. Symons, J. Chem. Soc. Faraday Trans. I 72, 2876 (1976).

[16] M. F. Ottaviani, J. Phys. Chem. 91, 779 (1987).

[17] C. Jolicoeur and H. L. Friedman, Ber. Bunsenges. Phys. Chem. 75, 248 (1971).

[18] D. Kivelson, J. Chem. Phys. 33, 1094 (1960). 